\title{
Filter games on $\omega$ and the dual ideal
}

\author{
by
}

\author{
Claude Laflamme (Calgary, AB) and \\ Christopher C. Leary (Geneseo, NY)
}

\begin{abstract}
We continue the efforts to characterize winning strategies in various infinite games involving filters on the natural numbers in terms of combinatorial or structural properties of the given filter. Previous results in the literature included those games where player II responded with natural numbers, or finite subsets of natural numbers. In this paper we concentrate on games where player II responds with members of the dual ideal. We also give a summary of known results on filter games.
\end{abstract}

\section{Introduction}

1.1. Overview of the problem. Combinatorial properties of filters have played an important role in applications of set theory, and the classical notions of a filter being meager, a P-filter or even selective have been around a long time. These concepts have been generalized in several ways in the literature, and we will concentrate on various tree and other structural properties. These combinatorial ideas have their roots in Ramsey theory where P-points and selective ultrafilters (sometimes called "Ramsey") have tree property characterizations. We refer the reader to early papers by Booth [4] and Grigorieff [7].

In this paper we pursue the investigations from [12] on filter games. Two players alternate turns in an $\omega$-sequence of innings, and typically, the games are played with respect to a fixed filter $\mathcal{F}$ on the natural numbers. Several objects are naturally related to a filter - the natural numbers themselves, the finite subsets of the natural numbers, the dual ideal $\mathcal{F}^{*}$ consisting of the complements of members in the filter, and finally $\mathcal{F}^{+}$containing subsets of natural numbers that can be safely added to the filter and still generate a proper filter. All these possibilities provide many versions of games, for example one which we label $\mathfrak{G}\left(\mathcal{F},[\omega]^{<\omega}, \mathcal{F}^{+}\right)$, where the first player succes-

2000 Mathematics Subject Classification: Primary 03E05; Secondary 03E15, 03E35.

This research was partially supported by NSERC of Canada. 
sively chooses members of $\mathcal{F}$, and the second player chooses a finite subset of that member; at the end of the game the second player is declared the winner if the union of his responses is a member of $\mathcal{F}^{+}$. The works [1], [2], [4], [6], [7], [12], [13], and [14] among others contain results on these and related filter games, and in this paper we concentrate on the mostly missing analysis when the second player responds with members of the dual ideal $\mathcal{F}^{*}$. We present a duality result for games of this sort, an apparently new flavor of filter combinatorics derived from one of these games, and finally some counterexamples used to differentiate various games. We also include a summary of known results on filter games.

Finally, we wish to warmly thank the anonymous referee for a thorough reading and very valuable comments for improving this paper.

1.2. Standard set-theoretic definitions. Our terminology is standard but we review the main concepts and notation. The set of natural numbers will be denoted by $\omega$, and $\wp(\omega)$ will denote the collection of all its subsets. Given $X \in \wp(\omega)$, we write $[X]^{\omega}$ and $[X]^{<\omega}$ to denote the collection of infinite or finite subsets of $X$ respectively. We use the well known "almost inclusion" ordering between members of $[\omega]^{\omega}$, i.e. $X \subseteq^{*} Y$ if $X \backslash Y$ is finite. A collection $\mathcal{X} \subseteq \wp(\omega)$ is said to be downward closed (under $\subseteq^{*}$ ) if $Y \subseteq^{*} X \in \mathcal{X}$ implies that $Y \in \mathcal{X}$.

We identify $\wp(\omega)$ with ${ }^{\omega_{2}}$ via characteristic functions. The space ${ }^{\omega_{2}}$ is further equipped with the product topology of the discrete space $\{0,1\}$. A basic neighborhood is then given by sets of the form

$$
\mathcal{O}_{s}=\left\{f \in \omega_{2}: s \subseteq f\right\}
$$

where $s \in{ }^{<} \omega_{2}$, the collection of finite binary sequences. The terms "nowhere dense", "meager", "Baire property" all refer to this topology. Concatenation of elements $\bar{s}, \bar{t} \in{ }^{<\omega} \omega$ will be written $\bar{s}^{\wedge} \bar{t}$.

A filter is a collection of subsets of $\omega$ closed under finite intersections, supersets and containing all cofinite sets; it is called proper if it contains only infinite sets.

Suppose that $\mathcal{X}$ is any collection of subsets of $\omega$. We denote by $\mathcal{X}^{+}$ the set $\left\{A \subseteq \omega: A^{\mathrm{c}} \notin \mathcal{X}\right\}$. Notice that $\mathcal{X}$ is upward closed if and only if $\mathcal{X}^{+}=\{Y \subseteq \omega: Y \cap X \neq \emptyset$ for all $X \in \mathcal{X}\}$.

Thus for a filter $\mathcal{F}, \mathcal{F}^{+}$denotes the collection of all sets $X$ such that $\langle\mathcal{F}, X\rangle$, the filter generated by $\mathcal{F}$ after adding $X$, is a proper filter; it is useful to notice that $X \in \mathcal{F}^{+}$if and only if $X^{\mathrm{c}} \notin \mathcal{F}$. $\mathcal{F}^{*}=\left\{X^{\mathrm{c}}: X \in \mathcal{F}\right\}$ is called the dual ideal, and equals $\left(\mathcal{F}^{+}\right)^{\mathrm{c}}=\wp(\omega) \backslash \mathcal{F}^{+}$, the collection of sets incompatible with $\mathcal{F}$. Thus $\mathcal{F}^{+}$is the co-ideal of $\mathcal{F}^{*}$, and so $\mathcal{F}^{+}$is also called the collection of $\mathcal{F}$-stationary sets. For convenience, we will use $\mathcal{F}^{\mathrm{c}}$ to denote $\wp(\omega) \backslash \mathcal{F}$. The Fréchet filter is the collection of cofinite sets, denoted by $\mathfrak{F} r$. 
The families $\mathcal{F}$ and $\mathcal{F}^{+}$are dual in a subtle sense: a set $X$ containing an element of each member of $\mathcal{F}$ (resp. $\mathcal{F}^{+}$) must belong to $\mathcal{F}^{+}$(resp. $\mathcal{F}$ ). In particular $\mathfrak{F} r$ and $[\omega]^{\omega}$ are dual in that sense. From more general work of Aczel [1] and Blass [3], there is a duality between games in which a player chooses $X_{k} \in \mathcal{F}$ while the other player responds with $n_{k} \in X_{k}$, and games in which a player chooses $Y_{k} \in \mathcal{F}^{+}$while the other player responds with $n_{k} \in Y_{k}$. The point is that the statements

$$
(\forall X \in \mathcal{F})(\exists n \in X)[\phi(n)] \quad \text { and } \quad\left(\exists Y \in \mathcal{F}^{+}\right)(\forall n \in Y)[\phi(n)]
$$

are equivalent.

1.3. Filter games. We will be interested in infinite filter games that we generally label $\mathfrak{G}(\mathcal{X}, \mathcal{Y}, \mathcal{Z})$, where $\mathcal{X}$ will usually be a filter $\mathcal{F}$ or a co-ideal $\mathcal{F}^{+}, \mathcal{Y}$ will be either $\omega,[\omega]^{<\omega}, \mathcal{F}^{*}$, or even $\mathcal{F}^{\mathrm{c}}$, and where $\mathcal{Z}$ will typically be $\mathcal{F}$ or $\mathcal{F}^{+}$. Other games with $\mathcal{Z}$ equal to $\mathcal{F}^{\mathrm{c}}$, or even $\mathcal{F}^{*}$, do arise but are mostly uninteresting.

The game $\mathfrak{G}(\mathcal{X}, \mathcal{Y}, \mathcal{Z})$ is played by two players $\mathbf{I}$ and $\mathbf{I I}$ as follows: in the $k$ th inning, where $k<\omega$, I chooses $X_{k} \in \mathcal{X}$, and then II responds with a non-empty subset $s_{k}$ of $X_{k}$ which is a member of $\mathcal{Y}$ (identify $\omega$ with $\{\{n\}: n \in \omega\}$ if $\mathcal{Y}=\omega)$. At the end of the game, II is declared the winner if $\bigcup_{k \in \omega} s_{k} \in \mathcal{Z}$.

1.4. Filter properties. We call a tree $\mathcal{T} \subseteq{ }^{<\omega} \omega$ an $\mathcal{X}$-tree for some $\mathcal{X} \subseteq$ $[\omega]^{\omega}$ ( $\mathcal{X}$ will usually be a filter $\mathcal{F}$ or $\mathcal{F}^{+}$) if for each $\bar{s} \in \mathcal{T}$, there is an $X_{\bar{s}} \in \overline{\mathcal{X}}$ such that $\bar{s}^{\wedge} n \in \mathcal{T}$ for all $n \in X_{\bar{s}}$. Similarly we call a tree $\mathcal{T} \subseteq{ }^{<\omega}\left([\omega]^{<\omega}\right)$ an $\mathcal{X}$-tree of finite sets for some $\mathcal{X} \subseteq[\omega]^{\omega}$, if for each $\bar{s} \in \mathcal{T}$, there is an $X_{\bar{s}} \in \mathcal{X}$ such that $\bar{s}^{\wedge} a \in \mathcal{T}$ for each $a \in\left[X_{\bar{s}}\right]^{<\omega}$. A branch of such a tree is thus an infinite sequence of finite sets and we will be interested in the union of such a branch, an infinite subset of $\omega$.

Here are the combinatorial properties of filters that have been (see [12]) or will be considered.

Definition 1. Let $\mathcal{F}$ be a filter on $\omega$.

(1) $\mathcal{F}$ is called a $Q$-filter if for any partition of $\omega$ into finite sets $\left\langle s_{k}\right.$ : $k \in \omega\rangle$, there is an $X \in \mathcal{F}$ such that $\left|X \cap s_{k}\right| \leq 1$ for all $k$.

(2) $\mathcal{F}$ is called a weak $Q$-filter if for any partition of $\omega$ into finite sets $\left\langle s_{k}: k \in \omega\right\rangle$, there is an $X \in \mathcal{F}^{+}$such that $\left|X \cap s_{k}\right| \leq 1$ for all $k$.

(3) $\mathcal{F}$ is called diagonalizable if there is an $X \in[\omega]^{\omega}$ such that $X \subseteq{ }^{*} Y$ for all $Y \in \mathcal{F}$.

(4) $\mathcal{F}$ is called $\omega$-diagonalizable if there are $\left\langle X_{n} \in[\omega]^{\omega}: n \in \omega\right\rangle$ such that for each $Y \in \mathcal{F}$, there is an $n$ such that $X_{n} \subseteq^{*} Y$.

(5) $\mathcal{F}$ is called $\omega$-+-diagonalizable if there are $\left\langle X_{n} \in \mathcal{F}^{+}: n \in \omega\right\rangle$ such that for each $Y \in \mathcal{F}$, there is an $n$ such that $X_{n} \subseteq^{*} Y$. 
(6) (a) A set $X \subseteq[\omega]^{<\omega}$ is called $\mathcal{Z}$-universal $\left(\mathcal{Z}\right.$ will be $\mathcal{F}$ or $\left.\mathcal{F}^{+}\right)$if for each $Y \in \mathcal{Z}$, there is an $x \in X \cap[Y]^{<\omega}$.

(b) $\mathcal{F}$ is called $\omega$-diagonalizable by $\mathcal{Z}$-universal sets if there are $\mathcal{Z}$-universal sets $\left\langle X_{n}: n \in \omega\right\rangle$ such that for all $Y \in \mathcal{F}$, there is an $n$ such that $x \cap Y \neq \emptyset$ for all but finitely many $x \in X_{n}$.

(7) $\mathcal{F}$ is a $P$-filter if given any sequence $\left\langle X_{n}: n \in \omega\right\rangle \subseteq \mathcal{F}$, there is an $X \in \mathcal{F}$ such that $X \subseteq^{*} X_{n}$ for each $n$.

(8) $\mathcal{F}$ is a $P^{+}$-filter if every $\mathcal{F}^{+}$-tree of finite sets has a branch whose union is in $\mathcal{F}^{+}$.

(9) $\mathcal{F}$ is a weak $P$-filter if given any sequence $\left\langle X_{n}: n \in \omega\right\rangle \subseteq \mathcal{F}$, there is an $X \in \mathcal{F}^{+}$such that $X \subseteq^{*} X_{n}$ for each $n$. Equivalently, every $\mathcal{F}$-tree of finite sets has a branch whose union is in $\mathcal{F}^{+}$.

(10) $\mathcal{F}$ is a P-point if it is an ultrafilter that is also a P-filter.

(11) $\mathcal{F}$ is Ramsey if any $\mathcal{F}$-tree has a branch in $\mathcal{F}$; equivalently, $\mathcal{F}$ is both a Q-filter and a P-filter.

(12) $\mathcal{F}$ is + -Ramsey if every $\mathcal{F}^{+}$-tree has a branch in $\mathcal{F}^{+}$.

(13) $\mathcal{F}$ is weakly Ramsey if any $\mathcal{F}$-tree has a branch in $\mathcal{F}^{+}$.

(14) $\mathcal{F}$ has the $P(\mathcal{X}, \mathcal{Y}, \mathcal{Z})$ property if

$$
\left(\forall Y_{0} \in \mathcal{Y}\right)\left(\exists X_{0} \in \mathcal{X}\right)\left(\forall Y_{1} \in \mathcal{Y}\right)\left(\exists X_{1} \in \mathcal{X}\right) \ldots\left[\bigcup_{n}\left(Y_{n} \backslash X_{n}\right) \in \mathcal{Z}\right] .
$$

In connection with this last definition, we will only be interested in filters with the $P\left(\mathcal{F}, \mathcal{F}, \mathcal{F}^{+}\right), P\left(\mathcal{F}, \mathcal{F}^{+}, \mathcal{F}^{+}\right)$, and $P\left(\mathcal{F}^{+}, \mathcal{F}, \mathcal{F}\right)$ properties. The property $P(\mathfrak{F} r, \mathcal{F}, \mathcal{F})$ is inconsistent with any filter. These notions do not seem to have appeared elsewhere as far as we know, and their relation with other properties will become more transparent later in the paper. For the moment, observe that the following variation in quantifier ordering:

$$
\left(\forall Y_{0} \in \mathcal{F}\right)\left(\forall Y_{1} \in \mathcal{F}\right) \ldots\left(\exists X_{0} \in \mathfrak{F} r\right)\left(\exists X_{1} \in \mathfrak{F} r\right) \ldots\left[\bigcup_{n}\left(Y_{n} \backslash X_{n}\right) \in \mathcal{F}\right]
$$

is equivalent to the P-filter property, and

$$
\left(\forall Y_{0} \in \mathcal{F}\right)\left(\forall Y_{1} \in \mathcal{F}\right) \ldots\left(\exists X_{0} \in \mathfrak{F} r\right)\left(\exists X_{1} \in \mathfrak{F} r\right) \ldots\left[\bigcup_{n}\left(Y_{n} \backslash X_{n}\right) \in \mathcal{F}^{+}\right]
$$

is equivalent to the weak P-filter property.

1.5. Earlier results. Games of the form $\mathfrak{G}(\mathcal{X}, \omega, \mathcal{Z})$ and even $\mathfrak{G}\left(\mathcal{X},[\omega]^{<\omega}, \mathcal{Z}\right)$ have been studied and combinatorial properties of winning strategies have been described in [2] and [12]. We remind the reader of two results of [12] that will be used in this paper.

Theorem 2. Fix a filter $\mathcal{F}$ and consider the game $\mathfrak{G}(\mathcal{F},[\omega]<\omega, \mathcal{F})$. Then

(1) I has no winning strategy if and only if $\mathcal{F}$ is a non-meager P-filter.

(2) II never has a winning strategy. 
TheOREM 3. Fix a filter $\mathcal{F}$ and consider the game $\mathfrak{G}\left(\mathcal{F},[\omega]^{<\omega}, \mathcal{F}^{+}\right)$. Then

(1) I has no winning strategy if and only if $\mathcal{F}$ is a weak P-filter.

(2) II has a winning strategy if and only if $\mathcal{F}$ is $\omega$-diagonalizable by $\mathcal{F}$-universal sets.

2. The ideal version and the duality theorem. Although recent research concerning these games has been cast in terms of filters, it will be convenient, and perhaps more natural, to notice that the same games can be described in terms of ideals.

2.1. Ideal game description. We consider an infinite ideal game labelled $\mathfrak{H}(\mathcal{I}, \mathcal{J}, \mathcal{Z})$, where $\mathcal{I}$ and $\mathcal{J}$ will be ideals and $\mathcal{Z}$ will usually be a filter or a co-ideal. The game $\mathfrak{H}(\mathcal{I}, \mathcal{J}, \mathcal{Z})$ is played by $\mathbf{I}$ and II in the following way: in inning $k$, with $k<\omega, \mathbf{I}$ chooses $I_{k} \in \mathcal{I}$ such that $I_{k}$ is disjoint from all previously played sets, and player II responds by selecting $J_{k} \in \mathcal{J}$ such that $J_{k}$ is also disjoint from all previously played sets. We will say that II wins a play of the game if $\bigcup_{k \in \omega} J_{k} \in \mathcal{Z}$. It is evident that $\mathfrak{G}(\mathcal{F}, \mathcal{J}, \mathcal{Z})$ is equivalent to $\mathfrak{H}\left(\mathcal{F}^{*}, \mathcal{J}, \mathcal{Z}\right)$ if $\mathcal{F}$ is a filter and $\mathcal{J}$ is an ideal - the only change being that in the $\mathfrak{H}$ game, I plays the complement of the set that she plays in the $\mathfrak{G}$ game. The motivation for the change is that in the ideal version, the game can be thought of as the two players claiming parts of $\omega$ for themselves, and II wins the game if the part that he claims is "large", where we think of $\mathcal{Z}$ as the collection of large sets.

2.2. The duality theorem. Recall that two games are called dual if a player has a winning strategy in one game iff the other player has a winning strategy in the other game. Results on dual games of course allow transfer principles to pass from one to the other.

Let us concentrate on games in which II is trying to construct a set in an upward closed family $\mathcal{Z}$. In this case, there is no loss of generality if we demand that the integer $k$ be chosen by one player or the other in inning $k$ of a play of the $\mathfrak{H}$ game. So in this case, II wins if he constructs a set in $\mathcal{Z}$, while $\mathbf{I}$ wins if she builds a set in $\mathcal{Z}^{+}$.

From this point of view, if we consider the two games $\mathfrak{H}(\mathcal{I}, \mathcal{J}, \mathcal{Z})$ and $\mathfrak{H}\left(\mathcal{J}, \mathcal{I}, \mathcal{Z}^{+}\right)$, the player playing elements of $\mathcal{I}$ has the same goal in each game. So does the player playing $\mathcal{J}$. Thus the only difference between the games is which player gets to move first. We will say that the two games $\mathfrak{H}(\mathcal{I}, \mathcal{J}, \mathcal{Z})$ and $\mathfrak{H}\left(\mathcal{J}, \mathcal{I}, \mathcal{Z}^{+}\right)$are paired. The duality theorem below says that paired games are dual if the families $\mathcal{Z}$ and $\mathcal{Z}^{+}$are large enough.

Definition 4. If $\mathcal{X}$ and $\mathcal{Y}$ are collections of subsets of $\omega$, we will say that $\mathcal{X}$ is $\mathcal{Y}$-robust if $X \backslash Y \in \mathcal{X}$ for every $X \in \mathcal{X}$ and every $Y \in \mathcal{Y}$. 
Notice that if $\mathcal{X}$ is upward closed, then $\mathcal{X}$ is $\mathcal{Y}$-robust if and only if $\mathcal{X}^{+}$ is $\mathcal{Y}$-robust.

Lemma 5. Suppose that player I has a winning strategy in $\mathfrak{H}(\mathcal{I}, \mathcal{J}, \mathcal{Z})$, and suppose that $\mathcal{Z}^{+}$is $\mathcal{J}$-robust. Then player $\mathbf{I}$ has a winning strategy for that game in which her first move is $\emptyset$.

Proof. Suppose $£$ is I's winning strategy. Consider a play of the game $\mathfrak{H}(\mathcal{I}, \mathcal{J}, \mathcal{Z}):$

\begin{tabular}{|c|c|c|c|c}
\hline Inning & 0 & 1 & 2 & $\ldots$ \\
\hline Player I & $\emptyset$ & $\left.£(\emptyset) \backslash J_{0}\right] \cup £\left(J_{0}\right)$ & $£\left(J_{0}, J_{1}\right)$ & $\ldots$ \\
\hline Player II & $J_{0}$ & $J_{1}$ & $J_{2}$ & $\ldots$ \\
\hline
\end{tabular}

Player I's payoff set after this play of the game is

$$
\left[£(\emptyset) \cup £\left(J_{0}\right) \cup £\left(J_{0}, J_{1}\right) \cup \ldots\right] \backslash J_{0},
$$

which is a set in $\mathcal{Z}^{+}$(as $£$ is a winning strategy) minus a set in $\mathcal{J}$. As $\mathcal{Z}^{+}$ is $\mathcal{J}$-robust, this payoff set is in $\mathcal{Z}^{+}$, and so $\mathbf{I}$ has won the game.

Corollary 6. Suppose that $\mathcal{Z}^{+}$is $\mathcal{J}$-robust and suppose that player $\mathbf{I}$ has a winning strategy in $\mathfrak{H}(\mathcal{I}, \mathcal{J}, \mathcal{Z})$. Then player $\mathbf{I I}$ has a winning strategy in $\mathfrak{H}\left(\mathcal{J}, \mathcal{I}, \mathcal{Z}^{+}\right)$.

Lemma 7. Suppose that player II has a winning strategy in $\mathfrak{H}(\mathcal{I}, \mathcal{J}, \mathcal{Z})$. Then player $\mathbf{I}$ has a winning strategy in $\mathfrak{H}\left(\mathcal{J}, \mathcal{I}, \mathcal{Z}^{+}\right)$.

Proof. For $\mathbf{I}$ to win $\mathfrak{H}\left(\mathcal{J}, \mathcal{I}, \mathcal{Z}^{+}\right)$, all she has to do is follow the winning strategy for $\mathbf{I I}$ in $\mathfrak{H}(\mathcal{I}, \mathcal{J}, \mathcal{Z})$, assuming that I's opening move in that game is $\emptyset$.

Theorem 8 (The duality theorem). Suppose that $\mathcal{I}$ and $\mathcal{J}$ are ideals, and suppose that $\mathcal{Z}$ is upward closed, and both $\mathcal{I}$ - and $\mathcal{J}$-robust. Then a player has a winning strategy in $\mathfrak{H}(\mathcal{I}, \mathcal{J}, \mathcal{Z})$ if and only if the other player has a winning strategy in $\mathfrak{H}\left(\mathcal{J}, \mathcal{I}, \mathcal{Z}^{+}\right)$. Thus the games $\mathfrak{H}(\mathcal{I}, \mathcal{J}, \mathcal{Z})$ and $\mathfrak{H}\left(\mathcal{J}, \mathcal{I}, \mathcal{Z}^{+}\right)$are dual.

Proof. Since $\left(\mathcal{Z}^{+}\right)^{+}=\mathcal{Z}$ and since $\mathcal{Z}$ is upward closed if and only if $\mathcal{Z}^{+}$is upward closed, the theorem follows immediately from Corollary 6 and from Lemma 7 as they show that a winning strategy for either player in $\mathfrak{H}(\mathcal{I}, \mathcal{J}, \mathcal{Z})$ implies a winning strategy for the opposite player in $\mathfrak{H}\left(\mathcal{J}, \mathcal{I}, \mathcal{Z}^{+}\right)$.

In applying the duality theorem, recall that (under the assumption that $\mathcal{Z}$ is upward closed) $\mathcal{Z}^{+}$is $\mathcal{J}$-robust if and only if $\mathcal{Z}$ is $\mathcal{J}$-robust. 
As we translate this result into the language of filters, let us extend the notion of robustness in the following way:

Definition 9. If $\mathcal{Z}$ is a collection of sets and $\mathcal{F}$ is a filter (resp. $\mathcal{F}^{+}$a co-ideal), we will say that $\mathcal{Z}$ is $\mathcal{F}$-robust (resp. $\mathcal{F}^{+}$-robust) if $Z \cap F \in \mathcal{Z}$ for all $Z \in \mathcal{Z}$ and all $F \in \mathcal{F}$ (resp. $\mathcal{F}^{+}$).

ThEOREM 10 (The duality theorem-filter version). Suppose that $\mathcal{F}$ and $\mathcal{G}$ are filters and suppose that $\mathcal{Z}$ is upward closed. Suppose also that $\mathcal{Z}$ is both $\mathcal{F}$ - and $\mathcal{G}$-robust. Then a player has a winning strategy in $\mathfrak{G}\left(\mathcal{F}, \mathcal{G}^{*}, \mathcal{Z}\right)$ if and only if the other player has a winning strategy in $\mathfrak{G}\left(\mathcal{G}, \mathcal{F}^{*}, \mathcal{Z}^{+}\right)$. Thus the games $\mathfrak{G}\left(\mathcal{F}, \mathcal{G}^{*}, \mathcal{Z}\right)$ and $\mathfrak{G}\left(\mathcal{G}, \mathcal{F}^{*}, \mathcal{Z}^{+}\right)$are dual.

EXAMPLE 11. The following are all examples of dual games, where $\mathcal{F}$ is any filter on $\omega$ :

(1) $\mathfrak{G}\left(\mathfrak{F} r,[\omega]^{<\omega}, \mathfrak{F} r\right)$ and $\mathfrak{G}\left(\mathfrak{F} r,[\omega]^{<\omega}, \mathfrak{F} r^{+}\right)$,

(2) $\mathfrak{G}\left(\mathcal{F}, \mathcal{F}^{*}, \mathcal{F}\right)$ and $\mathfrak{G}\left(\mathcal{F}, \mathcal{F}^{*}, \mathcal{F}^{+}\right)$,

(3) $\mathfrak{G}\left(\mathcal{F},[\omega]<\omega, \mathcal{F}^{+}\right)$and $\mathfrak{G}\left(\mathfrak{F} r, \mathcal{F}^{*}, \mathcal{F}\right)$,

(4) $\mathfrak{G}\left(\mathcal{F},[\omega]^{<\omega}, \mathcal{F}\right)$ and $\mathfrak{G}\left(\mathfrak{F} r, \mathcal{F}^{*}, \mathcal{F}^{+}\right)$.

3. Results that follow from the duality theorem. In this section we apply the duality theorem to games of the form $\mathfrak{G}\left(\mathcal{X}, \mathcal{F}^{*}, \mathcal{Z}\right)$, left entirely untouched in [12].

Corollary 12. Fix a filter $\mathcal{F}$ and consider the game $\mathfrak{G}\left(\mathfrak{F r}, \mathcal{F}^{*}, \mathcal{F}\right)$. Then

(1) $\mathbf{I}$ has a winning strategy if and only if $\mathcal{F}$ is $\omega$-diagonalizable by $\mathcal{F}$-universal sets.

(2) II has a winning strategy if and only if $\mathcal{F}$ is not a weak P-filter.

Proof. This is immediate from Theorem 3 , as $\mathfrak{G}\left(\mathfrak{F r}, \mathcal{F}^{*}, \mathcal{F}\right)$ is dual to $\mathfrak{G}\left(\mathcal{F},[\omega]<\omega, \mathcal{F}^{+}\right)$.

Corollary 13. Fix a filter $\mathcal{F}$ and consider the game $\mathfrak{G}\left(\mathfrak{F r}, \mathcal{F}^{*}, \mathcal{F}^{+}\right)$. Then

(1) I never has a winning strategy.

(2) II has a winning strategy if and only if $\mathcal{F}$ is meager or not a P-filter.

Proof. We notice that $\mathfrak{G}\left(\mathfrak{F} r, \mathcal{F}^{*}, \mathcal{F}^{+}\right)$is dual to $\mathfrak{G}(\mathcal{F},[\omega]<\omega, \mathcal{F})$, and then quote Theorem 2.

Proposition 14. Player I never has a winning strategy in $\mathfrak{G}\left(\mathcal{F}, \mathcal{F}^{*}, \mathcal{F}^{+}\right)$ and Player II never has a winning strategy in $\mathfrak{G}\left(\mathcal{F}, \mathcal{F}^{*}, \mathcal{F}\right)$.

Proof. Notice that the two games are dual, so I has a winning strategy in her game if and only if II has a winning strategy in his game. Assume either player has a winning strategy. Then they both do. Consider a play of 
a game where both players follow the winning strategy. At the end of the game, II has constructed a payoff set that must be an element of $\mathcal{F}$, as his strategy is a winning strategy. But as I's strategy is winning, that payoff set cannot be in $\mathcal{F}^{+}$, a contradiction.

4. Results that do not follow from the duality theorem. The following is a straightforward reformulation of the corresponding property.

Proposition 15. II has a winning strategy in the game $\mathfrak{G}\left(\mathcal{F}, \mathcal{F}^{*}, \mathcal{F}^{+}\right)$ if and only if $\mathcal{F}$ is $P\left(\mathcal{F}, \mathcal{F}, \mathcal{F}^{+}\right)$.

Corollary 16. I has a winning strategy in the game $\mathfrak{G}\left(\mathcal{F}, \mathcal{F}^{*}, \mathcal{F}\right)$ if and only if $\mathcal{F}$ is $P\left(\mathcal{F}, \mathcal{F}, \mathcal{F}^{+}\right)$.

Proof. This follows immediately from the previous proposition and the fact that $\mathfrak{G}\left(\mathcal{F}, \mathcal{F}^{*}, \mathcal{F}^{+}\right)$is paired with $\mathfrak{G}\left(\mathcal{F}, \mathcal{F}^{*}, \mathcal{F}\right)$.

Proposition 17. I has a winning strategy in the game $\mathfrak{G}\left(\mathcal{F}^{+}, \mathcal{F}^{*}, \mathcal{F}^{+}\right)$ if and only if $\mathcal{F}$ is $P\left(\mathcal{F}^{+}, \mathcal{F}, \mathcal{F}\right)$.

Proof. First, assume that $\mathcal{F}$ is $P\left(\mathcal{F}^{+}, \mathcal{F}, \mathcal{F}\right)$, and therefore we will use the fact that

$$
\left(\forall Y_{0} \in \mathcal{F}\right)\left(\exists X_{0} \in \mathcal{F}^{+}\right)\left(\forall Y_{1} \in \mathcal{F}\right)\left(\exists X_{1} \in \mathcal{F}^{+}\right) \ldots\left[\bigcup_{n}\left(Y_{n} \backslash X_{n}\right) \in \mathcal{F}\right] .
$$

We describe I's strategy as follows: I first puts $Y_{0}=\omega$ and uses $X_{0}$ from above as the first move.

In the $n$th inning, after II has responded with some $s_{k} \subseteq X_{k}, s_{k} \in \mathcal{F}^{*}$, then $\mathbf{I}$ defines $Y_{k+1}=s_{k}^{\mathrm{c}} \in \mathcal{F}$ and plays $X_{k+1}$ obtained from above.

At the end of the game, we must have $\bigcup_{n}\left(Y_{n} \backslash X_{n}\right) \in \mathcal{F}$ by assumption, and since $\bigcup_{k} s_{k}$ is disjoint from that set, we conclude that $\mathbf{I}$ has won the game.

Conversely, assume that $\mathbf{I}$ has a winning strategy $\$$ in $\mathfrak{G}\left(\mathcal{F}^{+}, \mathcal{F}^{*}, \mathcal{F}^{+}\right)$. We must show that $\mathcal{F}$ is $P\left(\mathcal{F}^{+}, \mathcal{F}, \mathcal{F}\right)$, i.e. that

$$
\left(\forall Y_{0} \in \mathcal{F}\right)\left(\exists X_{0} \in \mathcal{F}^{+}\right)\left(\forall Y_{1} \in \mathcal{F}\right)\left(\exists X_{1} \in \mathcal{F}^{+}\right) \ldots\left[\bigcup_{n}\left(Y_{n} \backslash X_{n}\right) \in \mathcal{F}\right] .
$$

Ignore the initial $Y_{0} \in \mathcal{F}$ for the moment, and let $X_{0}=\$(\emptyset)$. At the $k$ th inning, after we are given some $Y_{k+1} \in \mathcal{F}$, pretend that II responded with $a_{k}=\left(Y_{k+1}^{\mathrm{c}} \cap X_{k}\right) \in \mathcal{F}^{*}$ in the game $\mathfrak{G}\left(\mathcal{F}^{+}, \mathcal{F}^{*}, \mathcal{F}^{+}\right)$, and then let $X_{k+1}=$ $\$\left(a_{0}, a_{1}, \ldots, a_{k}\right)$.

Since $\$$ is a winning strategy for $\mathbf{I}$, we must have $\bigcup_{k} a_{k} \notin \mathcal{F}^{+}$, and therefore $Y_{0} \cap\left(\bigcup_{k} a_{k}\right)^{\mathrm{c}}=\bigcup_{k}\left(Y_{k} \backslash X_{k}\right) \in \mathcal{F}$ as required.

The following is again a simple reformulation of the corresponding property. 
Proposition 18. II has a winning strategy in the game $\mathfrak{G}\left(\mathcal{F}^{+}, \mathcal{F}^{*}, \mathcal{F}^{+}\right)$ if and only if $\mathcal{F}$ is $P\left(\mathcal{F}^{+}, \mathcal{F}, \mathcal{F}^{+}\right)$.

The following games are not paired in our sense, as $\mathcal{F}^{+}$is not $\mathcal{F}^{\mathrm{c}}$-robust, but are dual since strategies for either player are obvious.

Proposition 19. Player II always has a winning strategy in $\mathfrak{G}\left(\mathcal{F}, \mathcal{F}^{+}, \mathcal{F}\right)$ and player $\mathbf{I}$ always has a winning strategy in $\mathfrak{G}\left(\mathcal{F}^{\mathrm{c}}, \mathcal{F}^{*}, \mathcal{F}^{+}\right)$.

4.1. Winning strategies - summary and pairwise comparability. We summarize in Table 1 the various filter descriptions for winning strategies on filter games, including duality. These results can be found either in the current paper, or in [12] or else referenced in that paper.

Moreover, Figure 1 shows the various implications and non-implications between these properties, and hence between the corresponding winning strategies of filter games. The implications are almost all straightforward, either following from the definitions themselves, or through the winning strategy equivalence. One that remains is

Proposition 20. A filter is meager if and only if it is $\omega$-diagonalizable by $\mathfrak{F}$-universal sets.

Proof. We use a standard result of Talagrand [14] showing that a filter is meager if and only if there is a partition of $\omega$ into finite sets such that each member of the filter intersects all but finitely many elements of the partition.

Now suppose first that a filter $\mathcal{F}$ is $\omega$-diagonalizable by $\mathfrak{F} r$-universal sets, say $\left\langle X_{n}: n \in \omega\right\rangle$. Define a sequence of integers $\left\langle\pi_{n}: n \in \omega\right\rangle$ such that for each $n$, the interval $\left[\pi_{n}, \pi_{n+1}\right)$ contains an element of each $X_{i}$ as a subset, for $i \leq n$. Now given $Y \in \mathcal{F}$, by assumption there must be some $n$ such that almost all elements of $X_{n}$ intersect $Y$, and we conclude therefore that $Y$ intersects all but finitely many of the intervals $\left[\pi_{n}, \pi_{n+1}\right)$.

Conversely, assume that $\mathcal{F}$ is meager, and consider a partition $X$ of $\omega$ into finite sets such that each member of the filter intersects all but finitely many members of the partition. Then $X$ itself is $\mathfrak{F} r$-universal, and by the assumption it follows that $\mathcal{F}$ is $\omega$-diagonalizable by $X$ alone.

To show that the implications shown in Figure 2 are the only provable ones is more difficult. After the corresponding results in [12], it remains to consider those mostly concerned with the $P\left(\mathcal{F}, \mathcal{F}, \mathcal{F}^{+}\right)$property.

Although we do not have a structural description of filters with the various $P(\mathcal{X}, \mathcal{Y}, \mathcal{Z})$ properties, we have the following information concerning the games $\mathfrak{G}\left(\mathcal{F}, \mathcal{F}^{*}, \mathcal{F}^{+}\right)$and $\mathfrak{G}\left(\mathcal{F}^{+}, \mathcal{F}^{*}, \mathcal{F}^{+}\right)$, which do not fall under our duality result as $\mathcal{F}^{+}$is not $\mathcal{F}^{+}$-robust unless $\mathcal{F}$ is an ultrafilter. A similar result applies to $\mathcal{F}^{*}$ and $\mathcal{F}^{\mathrm{c}}$ in the case of an ultrafilter $\mathcal{F}$. 
Table 1. Duality and winning strategies for each player. "None" means "Not dual to any game $\mathfrak{G}(\mathcal{X}, \mathcal{Y}, \mathcal{Z}) "$.

\begin{tabular}{|c|c|c|c|}
\hline Game & Dual to game & $\begin{array}{l}\text { Filter property } \\
\text { for player I strategy }\end{array}$ & $\begin{array}{l}\text { Filter property } \\
\text { for player II strategy }\end{array}$ \\
\hline $\mathfrak{G}(\mathfrak{F} r, \omega, \mathcal{F})$ & $\mathfrak{G}\left(\mathfrak{F} r^{+}, \omega, \mathcal{F}^{\mathrm{c}}\right)$ & $\mathcal{F}$ not a Q-filter & Never \\
\hline $\mathfrak{G}\left(\mathfrak{F} r, \omega, \mathcal{F}^{+}\right)$ & $\mathfrak{G}\left(\mathfrak{F} r^{+}, \omega, \mathcal{F}^{*}\right)$ & $\mathcal{F}$ not a weak Q-filter & $\mathcal{F} \omega$-diagonalized \\
\hline $\mathfrak{G}\left(\mathfrak{F} r, \omega, \mathcal{F}^{\mathrm{C}}\right)$ & $\mathfrak{G}\left(\mathfrak{F} r^{+}, \omega, \mathcal{F}\right)$ & Never & Always \\
\hline $\mathfrak{G}\left(\mathfrak{F} r, \omega, \mathcal{F}^{*}\right)$ & $\mathfrak{G}\left(\mathfrak{F r}^{+}, \omega, \mathcal{F}^{+}\right)$ & $\mathcal{F}=\mathfrak{F} r$ & $\mathcal{F} \neq \mathfrak{F} r$ \\
\hline $\mathfrak{G}(\mathcal{F}, \omega, \mathcal{F})$ & $\mathfrak{G}\left(\mathcal{F}^{+}, \omega, \mathcal{F}^{\mathrm{c}}\right)$ & $\mathcal{F}$ not Ramsey & Never \\
\hline $\mathfrak{G}\left(\mathcal{F}, \omega, \mathcal{F}^{+}\right)$ & $\mathfrak{G}\left(\mathcal{F}^{+}, \omega, \mathcal{F}^{*}\right)$ & $\mathcal{F}$ not weakly Ramsey & $\mathcal{F} \omega$-+-diag. \\
\hline $\mathfrak{G}\left(\mathcal{F}, \omega, \mathcal{F}^{\mathrm{c}}\right)$ & $\mathfrak{G}\left(\mathcal{F}^{+}, \omega, \mathcal{F}\right)$ & Never & $\begin{array}{l}\mathcal{F} \text { not a Ramsey } \\
\text { ultrafilter }\end{array}$ \\
\hline $\mathfrak{G}\left(\mathcal{F}, \omega, \mathcal{F}^{*}\right)$ & $\mathfrak{G}\left(\mathcal{F}^{+}, \omega, \mathcal{F}^{+}\right)$ & $\mathcal{F}$ countably generated & $\mathcal{F}$ not + -Ramsey \\
\hline $\mathfrak{G}\left(\mathcal{F}^{+}, \omega, \mathcal{F}\right)$ & $\mathfrak{G}\left(\mathcal{F}, \omega, \mathcal{F}^{\mathrm{c}}\right)$ & $\begin{array}{l}\mathcal{F} \text { not a Ramsey } \\
\text { ultrafilter }\end{array}$ & Never \\
\hline $\mathfrak{G}\left(\mathcal{F}^{+}, \omega, \mathcal{F}^{+}\right)$ & $\mathfrak{G}\left(\mathcal{F}, \omega, \mathcal{F}^{*}\right)$ & $\mathcal{F}$ not +-Ramsey & $\mathcal{F}$ countably generated \\
\hline $\mathfrak{G}\left(\mathcal{F}^{+}, \omega, \mathcal{F}^{\mathrm{c}}\right)$ & $\mathfrak{G}(\mathcal{F}, \omega, \mathcal{F})$ & Never & $\mathcal{F}$ not Ramsey \\
\hline $\mathfrak{G}\left(\mathcal{F}^{+}, \omega, \mathcal{F}^{*}\right)$ & $\mathfrak{G}\left(\mathcal{F}, \omega, \mathcal{F}^{+}\right)$ & $\mathcal{F} \omega$-+-diag. & $\mathcal{F}$ not weakly Ramsey \\
\hline $\mathfrak{G}\left(\mathfrak{F} r,[\omega]^{<\omega}, \mathcal{F}\right)$ & $\mathfrak{G}\left(\mathfrak{F} r,[\omega]^{<\omega}, \mathcal{F}^{+}\right)$ & $\mathcal{F}$ meager & Never \\
\hline $\mathfrak{G}\left(\mathfrak{F} r,[\omega]^{<\omega}, \mathcal{F}^{+}\right)$ & $\mathfrak{G}\left(\mathfrak{F} r,[\omega]^{<\omega}, \mathcal{F}\right)$ & Never & $\mathcal{F}$ is meager \\
\hline $\mathfrak{G}\left(\mathfrak{F} r,[\omega]^{<\omega}, \mathcal{F}^{\mathrm{c}}\right)$ & $\mathfrak{G}\left(\mathfrak{F} r^{+}, \omega, \mathcal{F}\right)$ & Never & Always \\
\hline $\mathfrak{G}\left(\mathfrak{F} r,[\omega]^{<\omega}, \mathcal{F}^{*}\right)$ & $\mathfrak{G}\left(\mathfrak{F r}^{+}, \omega, \mathcal{F}^{+}\right)$ & $\mathcal{F}=\mathfrak{F} r$ & $\mathcal{F} \neq \mathfrak{F} r$ \\
\hline $\mathfrak{G}\left(\mathcal{F},[\omega]^{<\omega}, \mathcal{F}\right)$ & $\mathfrak{G}\left(\mathfrak{F} r, \mathcal{F}^{*}, \mathcal{F}^{+}\right)$ & $\begin{array}{l}\mathcal{F} \text { not a non-meager } \\
\text { P-filter }\end{array}$ & Never \\
\hline $\mathfrak{G}\left(\mathcal{F},[\omega]^{<\omega}, \mathcal{F}^{+}\right)$ & $\mathfrak{G}\left(\mathfrak{F} r, \mathcal{F}^{*}, \mathcal{F}\right)$ & $\mathcal{F}$ not a weak P-filter & $\begin{array}{l}\mathcal{F} \omega \text {-diag. } \\
\text { by } \mathcal{F} \text { univ. sets }\end{array}$ \\
\hline $\mathfrak{G}\left(\mathcal{F},[\omega]^{<\omega}, \mathcal{F}^{\mathrm{c}}\right)$ & $\mathfrak{G}\left(\mathcal{F}^{+}, \omega, \mathcal{F}\right)$ & Never & $\begin{array}{l}\mathcal{F} \text { not a Ramsey } \\
\text { ultrafilter }\end{array}$ \\
\hline $\mathfrak{G}\left(\mathcal{F},[\omega]^{<\omega}, \mathcal{F}^{*}\right)$ & $\mathfrak{G}\left(\mathcal{F}^{+}, \omega, \mathcal{F}^{+}\right)$ & $\mathcal{F}$ countably generated & $\mathcal{F}$ not +-Ramsey \\
\hline $\mathfrak{G}\left(\mathcal{F}^{+},[\omega]^{<\omega}, \mathcal{F}\right)$ & None & $\mathcal{F}$ not a P-point & Never \\
\hline $\mathfrak{G}\left(\mathcal{F}^{+},[\omega]^{<\omega}, \mathcal{F}^{+}\right)$ & None & $\mathcal{F}$ not a $\mathrm{P}^{+}$-filter & $\begin{array}{l}\mathcal{F} \omega \text {-diag. } \\
\text { by } \mathcal{F}^{+} \text {univ. sets }\end{array}$ \\
\hline $\mathfrak{G}\left(\mathcal{F}^{+},[\omega]^{<\omega}, \mathcal{F}^{\mathrm{c}}\right)$ & $\mathfrak{G}(\mathcal{F}, \omega, \mathcal{F})$ & Never & $\mathcal{F}$ not Ramsey \\
\hline $\mathfrak{G}\left(\mathcal{F}^{+},[\omega]^{<\omega}, \mathcal{F}^{*}\right)$ & $\mathfrak{G}\left(\mathcal{F}, \omega, \mathcal{F}^{+}\right)$ & $\mathcal{F} \omega$-+-diag. & $\mathcal{F}$ not weakly Ramsey \\
\hline $\mathfrak{G}\left(\mathfrak{F} r, \mathcal{F}^{*}, \mathcal{F}\right)$ & $\mathfrak{G}\left(\mathcal{F},[\omega]^{<\omega}, \mathcal{F}^{+}\right)$ & $\begin{array}{l}\mathcal{F} \omega \text {-diag. } \\
\text { by } \mathcal{F} \text { univ. sets }\end{array}$ & $\mathcal{F}$ not a weak P-filter \\
\hline $\mathfrak{G}\left(\mathfrak{F} r, \mathcal{F}^{*}, \mathcal{F}^{+}\right)$ & $\mathfrak{G}(\mathcal{F},[\omega]<\omega, \mathcal{F})$ & Never & $\begin{array}{l}\mathcal{F} \text { not a non-meager } \\
\text { P-filter }\end{array}$ \\
\hline $\mathfrak{G}\left(\mathfrak{F} r, \mathcal{F}^{*}, \mathcal{F}^{\mathrm{c}}\right)$ & $\mathfrak{G}\left(\mathfrak{F} r^{+}, \omega, \mathcal{F}\right)$ & Never & Always \\
\hline $\mathfrak{G}\left(\mathfrak{F} r, \mathcal{F}^{*}, \mathcal{F}^{*}\right)$ & $\mathfrak{G}\left(\mathfrak{F} r^{+}, \omega, \mathcal{F}\right)$ & Never & Always \\
\hline $\mathfrak{G}\left(\mathcal{F}, \mathcal{F}^{*}, \mathcal{F}\right)$ & $\mathfrak{G}\left(\mathcal{F}, \mathcal{F}^{*}, \mathcal{F}^{+}\right)$ & $P\left(\mathcal{F}, \mathcal{F}, \mathcal{F}^{+}\right)$ & Never \\
\hline $\mathfrak{G}\left(\mathcal{F}, \mathcal{F}^{*}, \mathcal{F}^{+}\right)$ & $\mathfrak{G}\left(\mathcal{F}, \mathcal{F}^{*}, \mathcal{F}\right)$ & Never & $P\left(\mathcal{F}, \mathcal{F}, \mathcal{F}^{+}\right)$ \\
\hline
\end{tabular}


Table 1 (cont.)

\begin{tabular}{|l|l|l|l|}
\hline Game & Dual to game & $\begin{array}{l}\text { Filter property } \\
\text { for player I strategy }\end{array}$ & $\begin{array}{l}\text { Filter property } \\
\text { for player II strategy }\end{array}$ \\
\hline $\mathfrak{G}\left(\mathcal{F}, \mathcal{F}^{*}, \mathcal{F}^{\mathrm{c}}\right)$ & $\mathfrak{G}\left(\mathfrak{F} r^{+}, \omega, \mathcal{F}\right)$ & Never & Always \\
$\mathfrak{G}\left(\mathcal{F}, \mathcal{F}^{*}, \mathcal{F}^{*}\right)$ & $\mathfrak{G}\left(\mathfrak{F} r^{+}, \omega, \mathcal{F}\right)$ & Never & Always \\
$\mathfrak{G}\left(\mathcal{F}^{+}, \mathcal{F}^{*}, \mathcal{F}\right)$ & None & $\mathcal{F}$ not an ultrafilter & Never \\
$\mathfrak{G}\left(\mathcal{F}^{+}, \mathcal{F}^{*}, \mathcal{F}^{+}\right)$ & None & $P\left(\mathcal{F}^{+}, \mathcal{F}, \mathcal{F}\right)$ & $P\left(\mathcal{F}^{+}, \mathcal{F}, \mathcal{F}^{+}\right)$ \\
$\mathfrak{G}\left(\mathcal{F}^{+}, \mathcal{F}^{*}, \mathcal{F}^{c}\right)$ & $\mathfrak{G}\left(\mathfrak{F} r^{+}, \omega, \mathcal{F}\right)$ & Never & Always \\
$\mathfrak{G}\left(\mathcal{F}^{+}, \mathcal{F}^{*}, \mathcal{F}^{*}\right)$ & $\mathfrak{G}\left(\mathfrak{F} r^{+}, \omega, \mathcal{F}\right)$ & Never & Always \\
\hline
\end{tabular}

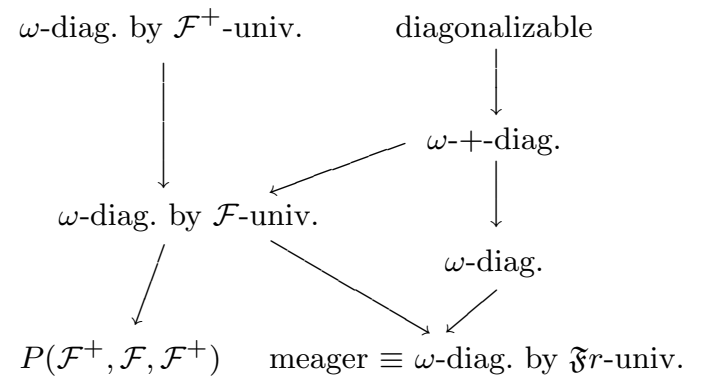

Fig. 1. Logical implications of the various filter properties

Proposition 21. If $\mathcal{F}$ is an ultrafilter, then neither player has a winning strategy in the games $\mathfrak{G}\left(\mathcal{F}, \mathcal{F}^{*}, \mathcal{F}^{+}\right)$and $\mathfrak{G}\left(\mathcal{F}^{+}, \mathcal{F}^{*}, \mathcal{F}^{+}\right)$.

Proof. If $\mathcal{F}$ is an ultrafilter, then $\mathcal{F}=\mathcal{F}^{+}$and the games $\mathfrak{G}\left(\mathcal{F}, \mathcal{F}^{*}, \mathcal{F}\right)$, $\mathfrak{G}\left(\mathcal{F}, \mathcal{F}^{*}, \mathcal{F}^{+}\right), \mathfrak{G}\left(\mathcal{F}^{+}, \mathcal{F}^{*}, \mathcal{F}\right)$, and $\mathfrak{G}\left(\mathcal{F}^{+}, \mathcal{F}^{*}, \mathcal{F}^{+}\right)$are all identical, and the result follows.

Although the two games $\mathfrak{G}\left(\mathcal{F}, \mathcal{F}^{*}, \mathcal{F}^{+}\right)$and $\mathfrak{G}\left(\mathcal{F}^{+}, \mathcal{F}^{*}, \mathcal{F}^{+}\right)$show similarities and are actually equivalent in case $\mathcal{F}$ is an ultrafilter, they are not equivalent in general as the following result shows.

Proposition 22. There is a $P\left(\mathcal{F}, \mathcal{F}, \mathcal{F}^{+}\right)$filter that is not $P\left(\mathcal{F}, \mathcal{F}^{+}, \mathcal{F}^{+}\right)$.

Proof. Let $\mathcal{F}=\bigotimes_{\omega} \mathfrak{F} r=\{A \subseteq \omega \times \omega:(\forall n)[\{m:(n, m) \in A\} \in \mathfrak{F} r\}]$. Then

$$
X \in \mathcal{F}^{+} \quad \text { if and only if } \quad(\exists n)[\{m:(n, m) \in X\} \text { is infinite }] .
$$

and

$$
X \in \mathcal{F}^{*} \quad \text { if and only if } \quad\left(\exists f \in{ }^{\omega} \omega\right)(\forall n)(\forall m)[(n, m) \in X \Rightarrow m \leq f(n)] .
$$

Therefore II has a winning strategy in $\mathfrak{G}\left(\mathcal{F}, \mathcal{F}^{*} \mathcal{F}^{+}\right)$by simply making sure to select infinitely many members of $\{(0, n): n \in \omega\}$. 
But $\mathbf{I}$ actually has a winning strategy in $\mathfrak{G}\left(\mathcal{F}^{+}, \mathcal{F}^{*} \mathcal{F}^{+}\right)$, simply by playing in the $n$th inning the set

$$
X_{n}=\{(n, m): m \in \omega\} \in \mathcal{F}^{+} .
$$

Player II has no choice but to choose only a finite subset of $X_{n}$, and at the end of the game the union of these replies is bounded by a fixed function, hence in $\mathcal{F}^{*}$.

Proposition 23. There is a non-meager $P\left(\mathcal{F}, \mathcal{F}^{+}, \mathcal{F}^{+}\right)$filter.

Proof. Let $\mathcal{U}$ be an ultrafilter and let

$$
\mathcal{F}=\bigotimes_{\mathfrak{F} r} \mathcal{U}=\left\{A \subseteq \omega \times \omega:\left(\forall^{\infty} n\right)[\{m:(n, m) \in A\} \in \mathcal{U}]\right\},
$$

a non-meager filter. We show II has a winning strategy in $\mathfrak{G}\left(\mathcal{F}^{+}, \mathcal{F}^{*}, \mathcal{F}^{+}\right)$. Observe that for $X \subseteq \omega \times \omega$,

$$
X \in \mathcal{F}^{+} \quad \text { if and only if } \quad\{n:\{m:(n, m) \in X\} \in \mathcal{U}\} \text { is infinite. }
$$

So at the $n$th inning, when I presents II with a set $X_{n} \in \mathcal{F}^{+}, \mathbf{I I}$ chooses $n$ such that $Y_{n}=\left\{m:(n, m) \in X_{n}\right\} \in \mathcal{U}$, and responds with $\{n\} \times Y_{n} \in \mathcal{F}^{*}$. At the end of the play, $Y=\bigcup_{n} Y_{n} \in \mathcal{F}^{+}$as infinitely many vertical sections are in $\mathcal{U}$.

Proposition 24. There is a $P\left(\mathcal{F}, \mathcal{F}^{+}, \mathcal{F}^{+}\right)$filter which is not a weak P-filter. Then

Proof. Let $\mathcal{F}=\bigotimes_{\mathfrak{F} r} \mathfrak{F} r=\left\{A \subseteq \omega \times \omega:\left(\forall^{\infty} n\right)[\{m:(n, m) \in A\} \in \mathfrak{F r}]\right\}$.

$$
X \in \mathcal{F}^{+} \quad \text { if and only if } \quad\{n:\{m:(n, m) \in X\} \text { is infinite }\} \text { is infinite. }
$$
and

$X \in \mathcal{F}^{*} \quad$ if and only if $\quad\left(\exists f \in{ }^{\omega} \omega\right)\left(\forall^{\infty} n\right)(\forall m)[(n, m) \in X \Rightarrow m \leq f(n)]$.

Therefore II has a winning strategy in $\mathfrak{G}\left(\mathcal{F}^{+}, \mathcal{F}^{*}, \mathcal{F}^{+}\right)$by making sure, at the $k$ th inning, to select an infinite subset of some $\{(n, m): m \in \omega\}$ for some $n>k$.

But II also has a winning strategy in $\mathfrak{G}\left(\mathfrak{F} r, \mathcal{F}^{*}, \mathcal{F}\right)$, simply by playing in the $n$th inning the set

$$
X_{n}=\{(n, m): m \in \omega\} \in \mathcal{F}^{*}
$$

intersected with I's move. Then at the end of the game, the union of II's replies contains all but finitely many elements of each $X_{n}$, and therefore is an element of $\mathcal{F}$.

The following should perhaps have been included in [12].

Proposition 25. There is a filter $\mathcal{F} \omega$-diagonalizable by $\mathcal{F}^{+}$-universal sets which is not a weak Q-filter. 
Proof. Fix a partition of $\omega$ into intervals $\left[\pi_{n}, \pi_{n+1}\right)$ such that $\pi_{n+1}-\pi_{n}=$ $n$ (or large enough). Let

$$
\mathcal{F}=\left\langle\left\{X^{\mathrm{c}}:(\forall n)\left[\left|X \cap\left[\pi_{n}, \pi_{n+1}\right)\right| \leq 1\right]\right\}\right\rangle .
$$

Clearly $\mathcal{F}$ is not a weak $Q$-filter by its very definition. However,

$$
X \in \mathcal{F}^{+} \quad \text { if and only if } \quad(\forall k)(\exists n)\left[\left|X \cap\left[\pi_{n}, \pi_{n+1}\right)\right| \geq k\right],
$$

and therefore II's winning strategy in $\mathfrak{G}\left(\mathcal{F}^{+},[\omega]^{<\omega}, \mathcal{F}^{+}\right)$is, after I's move $X$ at the $k$ th inning, to select $n$ large enough so that $X \cap\left[\pi_{n}, \pi_{n+1}\right)$ has more than $k$ elements and then reply with that set.

It is worth noticing that the $P\left(\mathcal{F}^{+}, \mathcal{F}, \mathcal{F}\right)$ does not appear in Figure 1. In fact, it is its negation that brings more interest, as it would be implied by the $\mathrm{P}^{+}$property. The fact that it is not entirely vacuous is shown by the following.

Proposition 26. There is a filter $\mathcal{F}$ with the $P\left(\mathcal{F}^{+}, \mathcal{F}, \mathcal{F}\right)$ property.

Proof. Let $\mathcal{F}=\bigotimes_{\omega} \mathfrak{F r}$. Then I wins $\mathfrak{G}\left(\mathcal{F}^{+}, \mathcal{F}^{*}, \mathcal{F}^{+}\right)$by blindly responding in the $k$ th inning with $X_{k}=\{(n, m): m \in \omega, n \geq k\}$.

At the end of the game, II's replies union to a subset of the plane bounded by a function, and therefore II's payoff set is in $\mathcal{F}^{*}$.

5. Conclusion and open questions. We know very little about games of the form $\mathfrak{G}\left(\mathcal{X}, \mathcal{F}^{\mathrm{c}}, \mathcal{Z}\right)$, but we have:

Proposition 27. In the game $\mathfrak{G}\left(\mathcal{F}, \mathcal{F}^{\mathrm{c}}, \mathcal{F}\right)$ :

(1) I never has a winning strategy.

(2) II has a winning strategy iff $\mathcal{F}$ is not an ultrafilter.

Proof. We first prove (2). Suppose that $\omega=A_{0} \cup A_{1}$ with $A_{i} \notin \mathcal{F}$. Player II's strategy can be played in 2 innings: II's response to I's first move $X_{0}$ is $X_{0} \cap A_{0} \notin \mathcal{F}$, and II's response to I's second move $X_{1}$ is $X_{1} \cap A_{1} \notin \mathcal{F}$. But

$$
\left(X_{0} \cap A_{0}\right) \cup\left(X_{1} \cap A_{1}\right) \supseteq X_{0} \cap X_{1} \in \mathcal{F} .
$$

Conversely, if $\mathcal{F}$ is an ultrafilter, then the game $\mathfrak{G}\left(\mathcal{F}, \mathcal{F}^{\mathrm{c}}, \mathcal{F}\right)$ is equivalent to both $\mathfrak{G}\left(\mathcal{F}, \mathcal{F}^{*}, \mathcal{F}\right)$ and $\mathfrak{G}\left(\mathcal{F}, \mathcal{F}^{*}, \mathcal{F}^{+}\right)$, but the last two games are dual to each other, and therefore II cannot have a winning strategy.

Finally, (1) follows from (2) and the previous paragraph.

Proposition 28. In the game $\mathfrak{G}\left(\mathfrak{F} r, \mathcal{F}^{\mathrm{c}}, \mathcal{F}\right)$ :

(1) II has a winning strategy iff $\mathcal{F}$ is not an ultrafilter.

(2) I has a winning strategy iff $\mathcal{F}$ is a P-point.

Proof. If $\mathcal{F}$ is not an ultrafilter, then II can follow a 2-inning strategy as in the previous proposition. 
Conversely, if $\mathcal{F}$ is an ultrafilter, then the game $\mathfrak{G}\left(\mathfrak{F} r, \mathcal{F}^{\mathrm{c}}, \mathcal{F}\right)$ is equivalent to $\mathfrak{G}\left(\mathfrak{F} r, \mathcal{F}^{*}, \mathcal{F}\right)$, which is dual to $\mathfrak{G}\left(\mathcal{F},[\omega]^{<\omega}, \mathcal{F}^{+}\right)$, which is finally equivalent to $\mathfrak{G}\left(\mathcal{F},[\omega]^{<\omega}, \mathcal{F}\right)$. Therefore by an earlier result quoted above, II does not have a winning strategy, and in this case $\mathbf{I}$ has a winning strategy iff $\mathcal{F}$ is a P-point.

We remark that these last results also prove that the games $\mathfrak{G}\left(\mathfrak{F} r, \mathcal{F}^{\mathrm{c}}, \mathcal{F}\right)$ and $\mathfrak{G}\left(\mathcal{F}^{+},[\omega]^{<\omega}, \mathcal{F}^{+}\right)$are not dual games.

A couple of natural variations on our game have yet to be examined. For example, a reasonable question to ask suggested by Eisworth [5] would be to see what happens if player I plays in one filter and player II plays in another filter. So the game might look something like $\mathfrak{G}\left(\mathcal{F}, \mathcal{G}^{+}, \mathcal{G}\right)$.

Another way to classify the games, suggested by Marion Scheepers, would be to extend the length of the game past an $\omega$ sequence of innings, and then to ask how long a play of the game would have to be to guarantee Player II a winning strategy.

Another possible variation is to play the game using filters on $\kappa$, where $\kappa$ is an uncountable cardinal, lengthening the play of the game as appropriate.

We have also been unsuccessful in our attempt to find nice structural properties of filters that are equivalent to the $P(\mathcal{X}, \mathcal{Y}, \mathcal{Z})$ property. We feel that is the largest open question concerning the pure filter game that we have investigated in this paper.

\section{References}

[1] P. Aczel, Quantifiers, games, and inductive definitions, in: Proc. Third Scandinavian Logic Symposium, S. Kanger (ed.), Stud. Logic Found. Math. 82, NorthHolland, 1975, 1-14.

[2] T. Bartoszyński and M. Scheepers, Filters and games, Proc. Amer. Math. Soc. 123 (1995), 2529-2534.

[3] A. Blass, Selective ultrafilters and homogeneity, Ann. Pure Appl. Logic 38 (1988), $215-255$.

[4] D. Booth, Ultrafilters on a countable set, Ann. Math. Logic 2 (1970), 1-24.

[5] T. Eisworth, Near coherence and filter games, Arch. Math. Logic 40 (2001), 235-242.

[6] F. Galvin, unpublished manuscript on ultrafilter games, $\approx 1980$.

[7] S. Grigorieff, Combinatorics on ideals and forcing, Ann. Math. Logic 3 (1971), 363-394.

[8] W. Just, A. R. D. Mathias, K. Prikry and P. Simon, On the existence of large $P$-ideals, J. Symbolic Logic 55 (1990), 457-465.

[9] K. Kunen, Some points in $\beta N$, Math. Proc. Cambridge Philos. Soc. 80 (1976), 385-398.

[10] C. Laflamme, Zapping small filters, Proc. Amer. Math. Soc. 114 (1992), 535-544.

[11] —, Strong meager properties for filters, Fund. Math. 146 (1995), 283-293.

[12] - Filter games and combinatorial properties of strategies, in: Contemp. Math. 192, Amer. Math. Soc., 1996, 51-67. 
[13] S. Shelah, Proper Forcing, Lecture Notes in Math. 940, Springer, Berlin, 1982.

[14] M. Talagrand, Compacts de fonctions mesurables et filtres non mesurables, Studia Math. 67 (1980), 13-43.

Department of Mathematics and Statistics

University of Calgary

Department of Mathematics

Calgary, Alberta

SUNY Geneseo

Canada T2N $1 \mathrm{~N} 4$

Geneseo, NY 14454, U.S.A.

E-mail: laf@math.ucalgary.ca

Received 10 November 2000;

in revised form 28 November 2001 